\title{
Standounkt
}

Grüne Gentechnik

\section{Prozent Ablehnung reichen nicht}

78 Prozent der Bundesbürger lehnen Gentechnik im Essen ab, so eine Forsa-Umfrage vom April diesen Jahres. Trotzdem fließen Millionen von Steuergeldern in diese Technik - mehr als in jede andere Sparte der Landwirtschaft. Dass öffentliche Meinung und politische Realität so auseinander klaffen, liegt auch an einem dichten Filz zwischen Konzernen, Behörden, Forschung und Lobbyisten. Von Jörg Bergstedt

m Juni wurden die neuen Mitglieder der europäischen Kontrollbehörde für Gentechnik ernannt (1). Aus Deutschland werden vier Experten in das Gremium entsandt. Sie alle sind uneingeschränkte Befürworter(innen) der Gentechnik. Das ist keine Ausnahme, denn alle relevanten Posten bei Geldvergabe- und Kontrollstellen sind mit Anhängern dieser Technologie besetzt (2). Etliche Bundesbehörden forschen und entwickeln selbst mit.

Noch dramatischer ist die Lage in internationaler Perspektive. Dort vergrößern Vertreibung, Umweltzerstörung und die Zerschlagung lokaler Märkte den Hunger. Dennoch forcieren die Industrienationen mit sogenannten Hilfsprogrammen ausgerechnet die Abhängigkeit erhöhende Gentechnologie. Es geht nur um Macht und Profit, während scheinheilig eine sachliche Debatte eingefordert wird.

\section{Ein Blick in die Amtsstuben}

Dabei zeigt schon der Blick in die Amtsstuben der Entscheidungsträger, dass die Sache dort längst geklärt ist. Es wird gehandelt. Die wichtigsten Beamten der zuständigen Genehmigungsbehörde, dem Bundesamt für Verbraucherschutz und Lebensmittelsicherheit, sind ebenso wie die sie beratenden Fachgremien und Bundesanstalten direkt in die Geflechte von Gentechnikkonzernen und Biotechnologie-Kleinstfirmen eingebunden (2). Dass Anträge auf Freisetzungen manipulierter Pflanzen politisch und amtlich unterstützt, also durchgewunken, mit so- fortiger Vollziehung gegen Bürgereinwendungen abgesichert und finanziert werden, ist vor diesem Hintergrund nicht überraschend.

Noch schlimmer sind die quasi mafiösen Geflechte von Kleinstunternehmen, staatlichen Geldgebern, vermeintlich unabhängigen Forscher(inn)en und Kontrollinstitutionen an zwei Orten riskanter Vielfachfreisetzungen. In der Biotechfarm in Üplingen (Börde) und im Agrobiotechnikum in Groß Lüsewitz (nahe Rostock) werden große Mengen von Fördergeldern hin- und hergeschoben, bis sich ihre Spur auf den Konten der Beteiligten verliert (2). Sicherheitsauflagen werden ständig missachtet und Versuchsziele in Anträgen falsch angegeben, um die Fördermittel zu erhalten. Kein Wunder, dass die Protagonisten möglichst wenig auffallen wollen. Die Devise ist: Wegducken und einfach weitermachen. BASF, Monsanto, Bayer, KWS und andere lassen Kleinstfirmen die Arbeit machen, staatliche Stellen verweigern, widerrechtlich, die Akteneinsicht nach dem Umweltinformationsgesetz (3).

\section{Fakten schaffen bevor sich die Gesetzeslage ändert}

Dabei wissen diejenigen, die am Ende den Profit einstreichen, dass sie gewinnen, wenn sie es weiter schaffen, gentechnisch veränderte Organismen in die Umwelt $\mathrm{zu}$ bringen. Auch kleine Felder bringen viel. In Deutschland gab es um die Jahrtausendwende etliche Forschungsfelder mit Raps. Sie waren nie groß, aber gereicht hat es, dass das gentechnische Material in den Regalen der Lebensmittelläden zu finden ist. Die Ausbreitung von Gentechnik ist als natürlicher Vorgang so intensiv und unumkehrbar, dass es keine Gentechnikfreiheit mehr gibt, wenn nur ausreichend viele Quellen der Auskreuzung etabliert werden können. Die Unterstützung williger Beamter in staatlichen Stellen ist sicher. Daran ändert die Einzelentscheidung zum Verbot der gentechnisch veränderten Maissorte MON810 der US-amerikanischen Konkurrenz wenig.

Pollen, Bienen und gentransferierende Mikroorganismen interessieren sich weder für Parlamente noch für Gesetzestexte oder Kaufentscheidungen. Ob Gentechnikfreiheit möglich ist, entscheidet sich nicht am Ladenregal, sondern dort, wo die Felder angelegt werden - und damit, dass der Filz in Behörden, Fachanstalten und Forschung gründlich ausgemistet wird. Genau davon lenken nicht nur Gentechnikfirmen ab, sondern auch Verbraucher- und Umweltschutzorganisationen mit ihrer Suggestion der Verbrauchermacht, die es hier nicht gibt.

\section{Anmerkungen}

(1) Im Internet unter:

http://www.biosicherheit.de/de/aktuell/694.do ku.html

(2) Genauere Quellen, weitere Informationen und Beispielfälle finden sich in: Bergstedt, Jörg: „Organisierte Unverantwortlichkeit“. Seiten Hieb Verlag, Reiskirchen 2009.

(3) Im Internet unter: http://www.projektwerkstatt.de/gen/sonder_bvl_akteneinsicht.htm

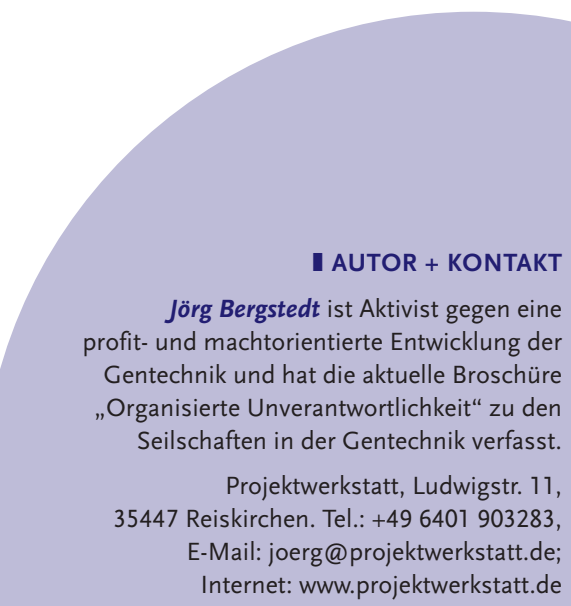


(c) 20I0 Authors; licensee IÖW and oekom verlag. This is an article distributed under the terms of the Creative Commons Attribution Non-Commercial No Derivates License (http://creativecommons.org/licenses/by-nc-nd/3.o/), which permits unrestricted use, distribution, and reproduction in any medium, provided the original work is properly cited. 